\title{
Analyse de risques pour les circulations ferroviaires d'un débordement de drainage sur ligne à grande vitesse (LGV)
}

\author{
Cicely PAMS CAPOCCIONI ${ }^{1}$, Damien NIVON² , Julie AMBLARD ${ }^{3}$, Giovanni DE CESARE ${ }^{4}$, Tamara GHILARDI ${ }^{5}$ \\ SNCF RESEAU, Direction Ingenierie et projet - 6, avenue François Mitterrand 93574 LA PLAINE SAINT DENIS \\ 1. cicely.pams@sncf.fr \\ 2.damien.nivon@sncf.fr \\ 3.julie.amblard@sncf.fr \\ EPFL Laboratoire de Constructions Hydrauliques - CH - 1015 LAUSANNE \\ 4. giovanni.decesare@epfl.ch \\ 5. tamara.ghilardi@epfl.ch
}

RÉSUMÉ. - Suite à un incident survenu en 2000 sur la Ligne à Grande Vitesse (LGV) Paris-Lyon sur la commune de Sarry dû à un emportement de ballast par débordement des eaux du drainage longitudinal de la plateforme ferroviaire, la SNCF a engagé en tant que gestionnaire délégué de l'infrastructure un ensemble de démarches afin de mieux caractériser le « risque Sarry ». Une campagne d’expertise a été ainsi initiée sur les LGV les plus anciennes afin de repérer les sites à risque de débordement de drainage pouvant impacter les circulations ferroviaires. Par la suite, des travaux de régénération ont été réalisés sur les sites priorisés les plus à risque afin d’éliminer le risque hydraulique. En parallèle, la LGV Paris-Marseille est surveillée en permanence par des radars météorologiques pour permettre le déclenchement d’alertes en cas de dépassement de seuils pluviométriques. Ce déclenchement permet l'avertissement du centre de régulation des trains en contact avec les conducteurs de TGV et entraîne une tournée intempéries par les agents de maintenance. Des recherches ont été menées en collaboration avec l'Ecole Polytechnique Fédérale de Lausanne (EPFL) avec la réalisation d'un modèle physique à l'échelle 1/3. Les résultats ont permis de mieux appréhender le phénomène de transport de ballast et d'identifier les limites d'emportement de ballast sur piste pour les deux critères de détermination du risque : la hauteur d'eau et la vitesse de l'écoulement.

Ces démarches établies à différentes échelles permettent de réduire la vulnérabilité des circulations ferroviaires vis-à-vis du risque de débordement de drainage, appelé « risque Sarry ».

Mots-clés : risque, débordement, modèle physique, ferroviaire

\section{Analysis of the risks for rail traffic in the event of overflowing of the drainage system on high speed lines}

Following an incident at the village of Sarry in 2000 involving the overflowing of the longitudinal drains of the Paris-Lyon High-Speed Line (HSL), the SNCF, responsible for the maintenance of the infrastructure, outlined a wide range of initiatives to define the "Sarry risk". A campaign of visits by experts has been initiated on the oldest HSLs in order to detect the sites at risk of overflowing and which could impact the railway traffic. In areas classified as "high risk areas”, regeneration works are undertaken to eliminate the risk. At the same time, the HSL between Paris and Marseille is monitored permanently by meteorological radars with a view to issuing alerts in case of exceedance of pluviometrical thresholds. This alert allows the railway regulation centre, in contact with the HSL drivers to be kept informed and results in a surveillance visit by the maintenance staff. Research has been undertaken in collaboration with EPFL (Ecole Polytechnique Fédérale de Lausanne) to realise a physical model at 1/3 scale. Results are used to better understand the phenomena of ballast loss and identify limits of ballast removal for two criteria : water level and flow velocity which are the criteria used in determining the level of risk.

This approach at different scales aims to reduce the vulnerability of railway traffic caused by the overflowing of the adjacent hydraulic structures.

Key-words: risk, overflowing, physical model, railway

\section{INTRODUCTION}

Un incident survenu en 2000 sur la ligne Paris-Lyon a entraîné un emportement de ballast dû au débordement du drainage longitudinal de la plate-forme ferroviaire qui a nécessité l'arrêt des circulations. La SNCF a engagé en tant que gestionnaire délégué de l'infrastructure un ensemble de démarches afin de mieux caractériser le
« risque Sarry » pour comprendre le phénomène physique d'emportement de la banquette de ballast et mesurer l'ampleur de la problématique sur l'ensemble des Lignes à Grande Vitesse (LGV) existantes [Nivon et Pams Capoccioni, 2011 ; Pams Capoccioni et al., 2012 ; Pams Capoccioni et al., 2013].

Les démarches entreprises sont de plusieurs ordres et vont de la prévention du risque à la suppression de 
celui-ci sur les sites les plus exposés. Parmi les démarches entreprises, la plus représentative du lien conceptionmaintenance-recherche de l'Ingénierie SNCF est sans aucun doute celle de réaliser des essais sur modèle physique afin d'améliorer la connaissance du risque.

\section{CONTEXTE DE L'EVENEMENT DE SARRY}

Suite à un orage court et intense, sur la commune de Sarry dans l'Yonne (cf. Figure 1) le 12 septembre 2000, la pluie mêlée à la grêle, d'une rare violence a provoqué un ruissellement considérable, entraînant des coulées de boue importantes dans le déblai de la ligne à grande vitesse Paris Sud Est (PSE).

L'eau mélangée à du transport solide a été collectée par les fossés en pied de déblai (cf. Figure 2). Toutefois, à cause d'une capacité insuffisante des drainages, encore aggravée par des dispositifs bouchés par la boue, l'excédent d'écoulement a débordé sur la piste emportant le pied de banquette de ballast.

Puis les matériaux transportés ont bouché la buse sous le pont-route du Point Kilométrique (PK) 164+591 (cf. Figure 2), ce qui a augmenté l'écoulement en piste qui, en plus, a pris de la vitesse sous le pont-route du fait du rétrécissement de la piste sous l'ouvrage d'art. Il est important de noter qu'à cet endroit la pente longitudinale de la piste est de $3,5 \%$.
L'eau a ensuite emporté le ballast sous les traverses, en traversant les voies de V2 vers V1 (dévers de plateforme à cet endroit). La circulation a été interrompue suite à l'alerte d'un choc anormal par le mécanicien d'un train circulant sur la voie opposée.

\section{LES DEMARCHES ENTREPRISES SUITE À L'EVENEMENT}

La SNCF a entrepris différentes actions dans le but de limiter les risques vis-à-vis de la sécurité des circulations ferroviaires sur l'ensemble du réseau ferré à grande vitesse.

Le terme " risque Sarry " a été défini comme le risque de débordement des dispositifs de drainage existants pouvant provoquer un entraînement du ballast, susceptible d'impacter les circulations ferroviaires.

Recenser et hiérarchiser le risque sur LGV

La première démarche entreprise fut l'expertise de l'ensemble du réseau hydraulique sur les Lignes à Grande Vitesse afin d'effectuer un diagnostic global des drainages existants et de connaître leur état.

Le "risque Sarry » est directement lié aux règles de dimensionnement et de conception adoptées lors de la construction des lignes nouvelles et aux éventuelles modifications apportées sur le réseau de drainage (diminution du débouché hydraulique ou discontinuité des installations existantes).

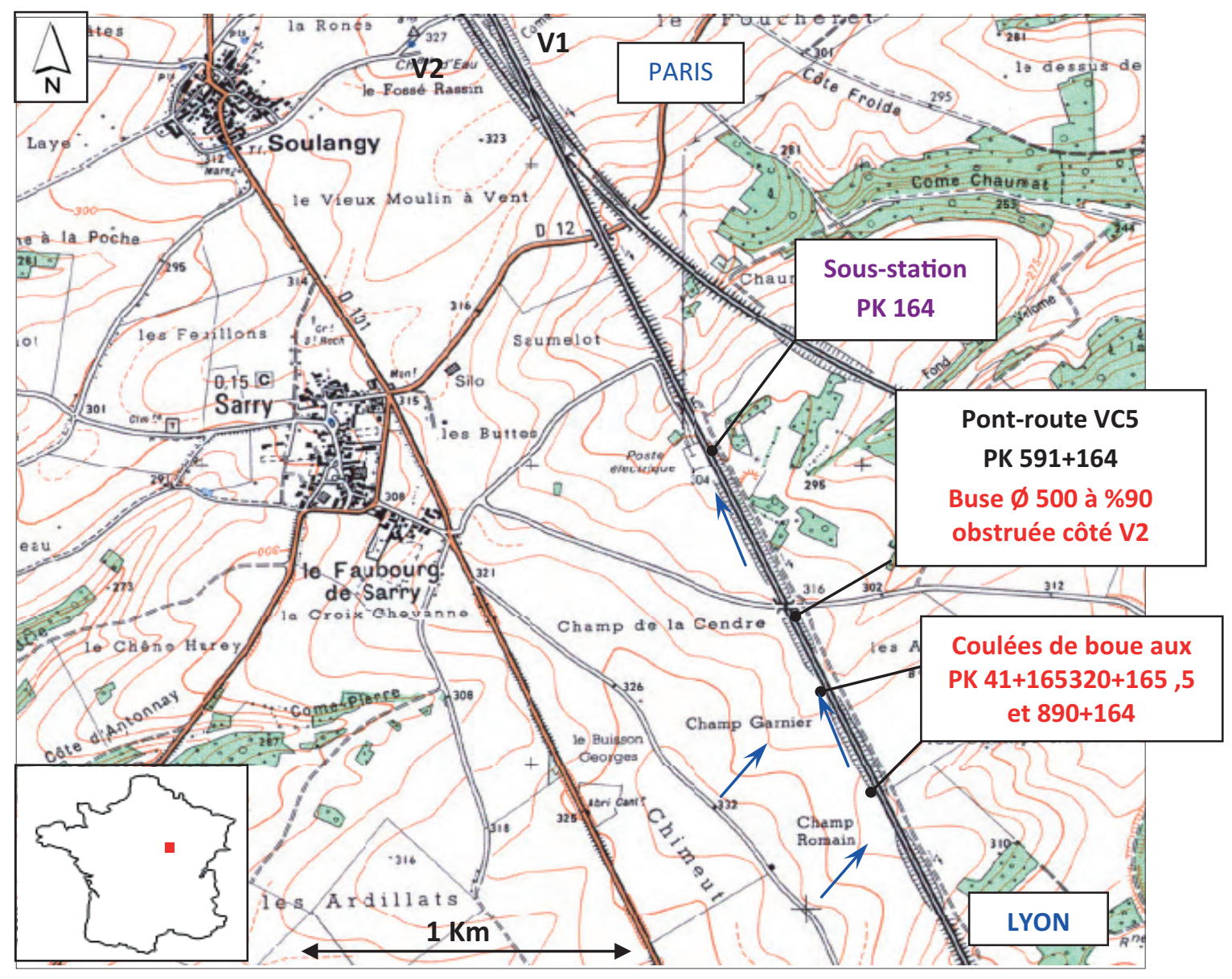

Figure 1 : Plan de situation de l'incident de Sarry, 12 septembre 2000. 

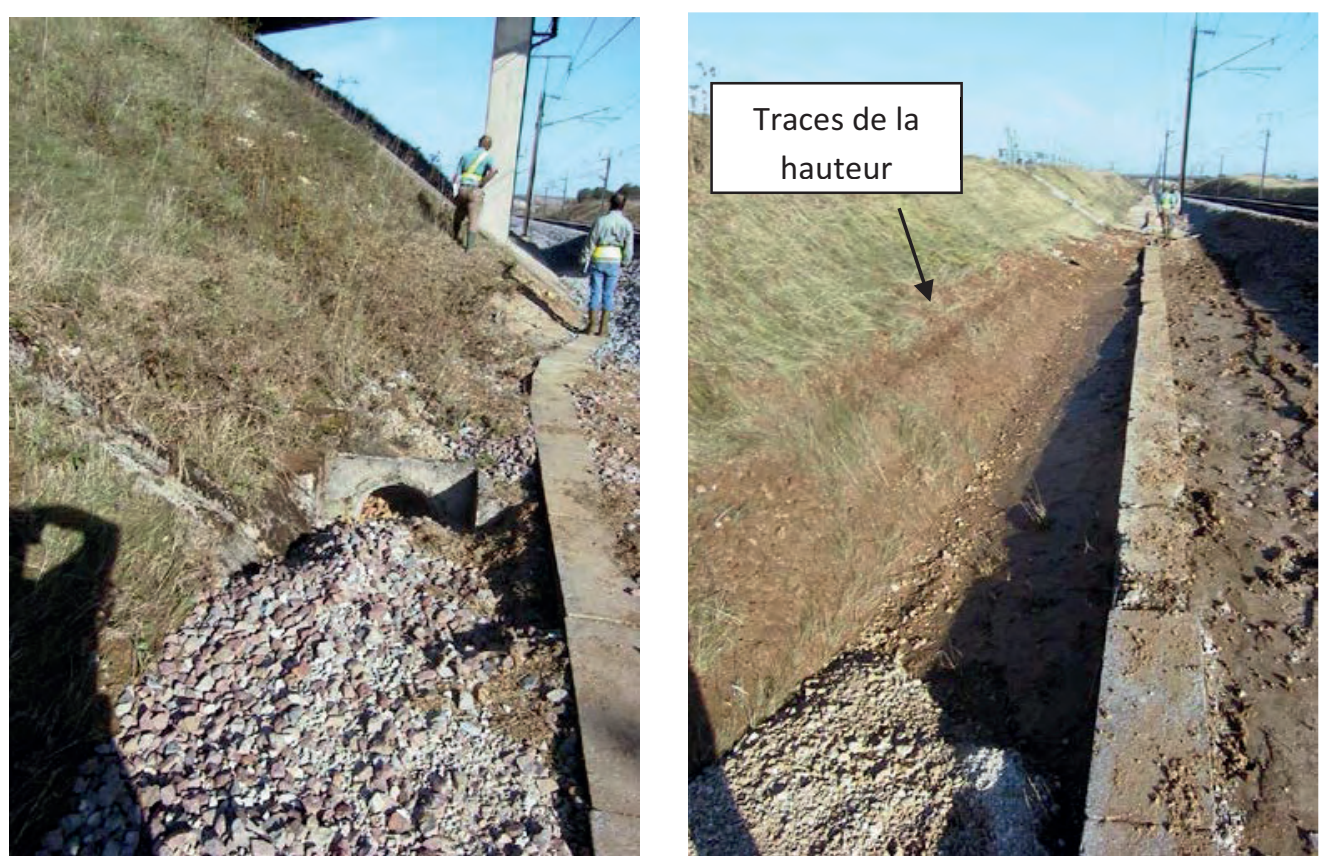

Figure 2 : Dégâts causés côté V2 (gauche) et V1 (droite).

L'identification des sites a été effectuée en réalisant une analyse de la vulnérabilité de l'infrastructure liée aux risques de débordement. Pour chacune des lignes visitées, les expertises aboutissent à un classement des sites visités en trois catégories suivant les critères suivants :

> Catégorie 1 : Sites présentant un dimensionnement hydraulique insuffisant et un risque préjudiciable pour les circulations lors d'un événement pluvieux inférieur au temps de retour de conception ;

$>$ Catégorie 2 : Sites présentant un dimensionnement hydraulique insuffisant mais un risque non préjudiciable pour les circulations jusque pour un temps de retour de conception ;

$>$ Catégorie 3 : Sites potentiellement vulnérables mais sans risque pour les circulations jusqu'au temps de retour de conception.

Les résultats des expertises menées sur les Lignes à Grande Vitesse françaises montrent clairement une diminution des sites classés en catégorie 1 (les plus à risque) en adéquation avec l'évolution des règles de conception depuis la mise en service de la LGV PSE dans les années 80 (cf. Tableau 1).

Lors de la construction de nouvelles LGV, le référentiel technique pour la réalisation des LGV permet de concevoir et de réaliser les ouvrages de génie civil de l'infrastructure. Il énonce entre autres les règles de dimensionnement relatives aux ouvrages hydrauliques (maintenance et sécurité). Après chaque projet de construction d'une ligne nouvelle, ce référentiel a été adapté et donc modifié.

Le référentiel technique actuel impose le dimensionnement du drainage longitudinal des voies au débit décennal et le dimensionnement des ouvrages hydrauliques de traversée sous voie au débit centennal. Il prend également en compte la problématique liée au « risque Sarry » puisque le comportement des ouvrages hydrauliques doit être décrit au niveau des singularités (transition fossé / buse par exemple) pour les débits extrêmes $\left(1.8 \times \mathrm{Q}_{\text {projet }}\right)$ afin d'éviter le phénomène de débordement en piste ou de submersion des voies.
Le paramètre multiplicateur d'1.8 a été déterminé arbitrairement (à dires d'expert), il permet l'analyse du risque hydraulique au niveau de ces points particuliers (ouvrages sous pont-route, descentes d'eau, etc.).

\section{III.1. Supprimer le risque sur les sites les plus exposés}

Un programme de régénération des ouvrages hydrauliques a été lancé permettant ainsi de supprimer le risque sur les sites les plus exposés. Cette campagne consiste à la réalisation de travaux d'hydraulique (bassins de rétention, redimensionnement des ouvrages, etc.) suite à une analyse de risque précise de la zone.

En hydraulique, les critères usuels pour caractériser un écoulement que ce soit en rivière, dans un tuyau ou dans un canal, sont la hauteur d'eau et la vitesse d'écoulement. Ces valeurs permettent ensuite d'appréhender des notions de débit, d'érosion et d'énergie.

En ce qui concerne le « risque Sarry ", il s'agit de caractériser l'écoulement sur la piste notamment en pied de la banquette de ballast. Par conséquent, pour obtenir les valeurs de hauteur et de vitesse sur la piste, il est nécessaire de mener une étude hydraulique complète avec modélisation mathématique des écoulements. Pour cela, il faut connaître exactement la topographie de la zone où l'eau pourrait s'écouler. Des dizaines de profils en travers et de nuages de points peuvent être nécessaires. Les résultats de cette modélisation permettent de connaître en tout point de la zone inondée, la hauteur et la vitesse par l'intermédiaire de profil en travers (cf. Figure 3).

Le diagnostic mené via la modélisation numérique permet ainsi d'identifier les zones à risque avec précision et ensuite proposer des aménagements dimensionnés permettant d'annuler le risque initialement présent. Cette campagne de régénération menée depuis 10 ans a permis la remise à niveau de dizaines de points singuliers jugés les plus à risque (cf. Figure 4). 
Tableau 1 : Evolution des règles de dimensionnement au cours des constructions des lignes à grande vitesse (Perlet, 2002).

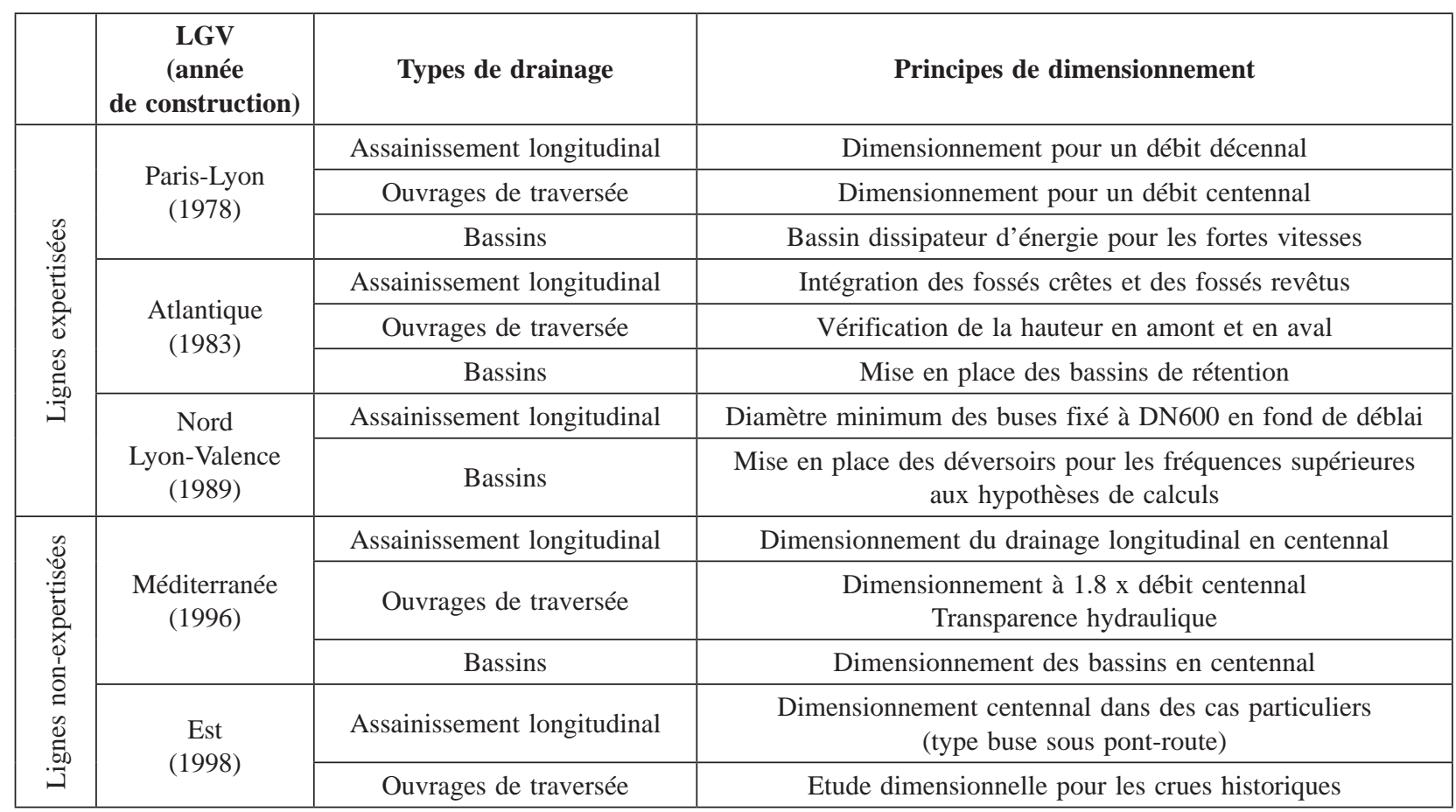

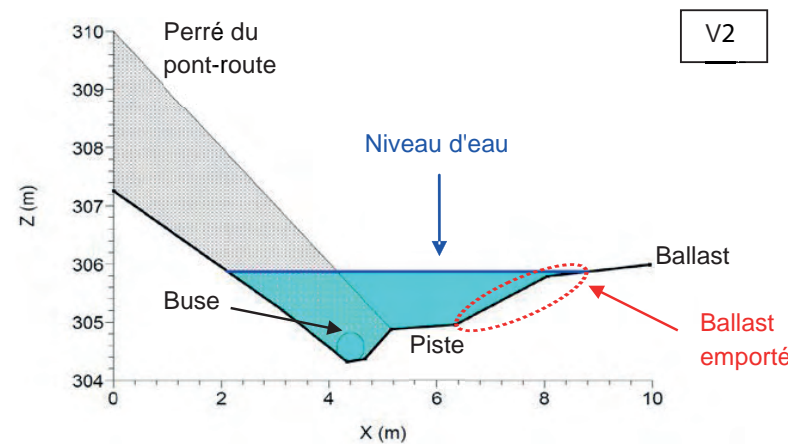

Figure 3 : Profil en travers obtenu avec une modélisation hydraulique pour $Q_{10}$ (cas du pont-route à Sarry).

\section{III.2. Vigilance météo pour surveiller la LGV}

La LGV PSE est surveillée depuis 1998 par des radars météorologiques sur l'ensemble de son linéaire. Suite à la mise en service de la LGV Méditerranée (2001), la surveillance de la ligne a été prolongée sur l'ensemble du linéaire de Paris à Marseille. Le déclenchement des alertes (cf. Figure 5) se fait lorsque la hauteur de pluie en un temps donné dépasse les seuils fixés. Ce processus d’alerte effectué par un prestataire météorologique permet de prévenir le centre de supervision des circulations ainsi que les établissements de maintenance, lorsqu'un orage important a lieu sur la ligne.

Le centre de supervision peut ainsi décider de prévenir les conducteurs des TGV présents dans la zone et demander un
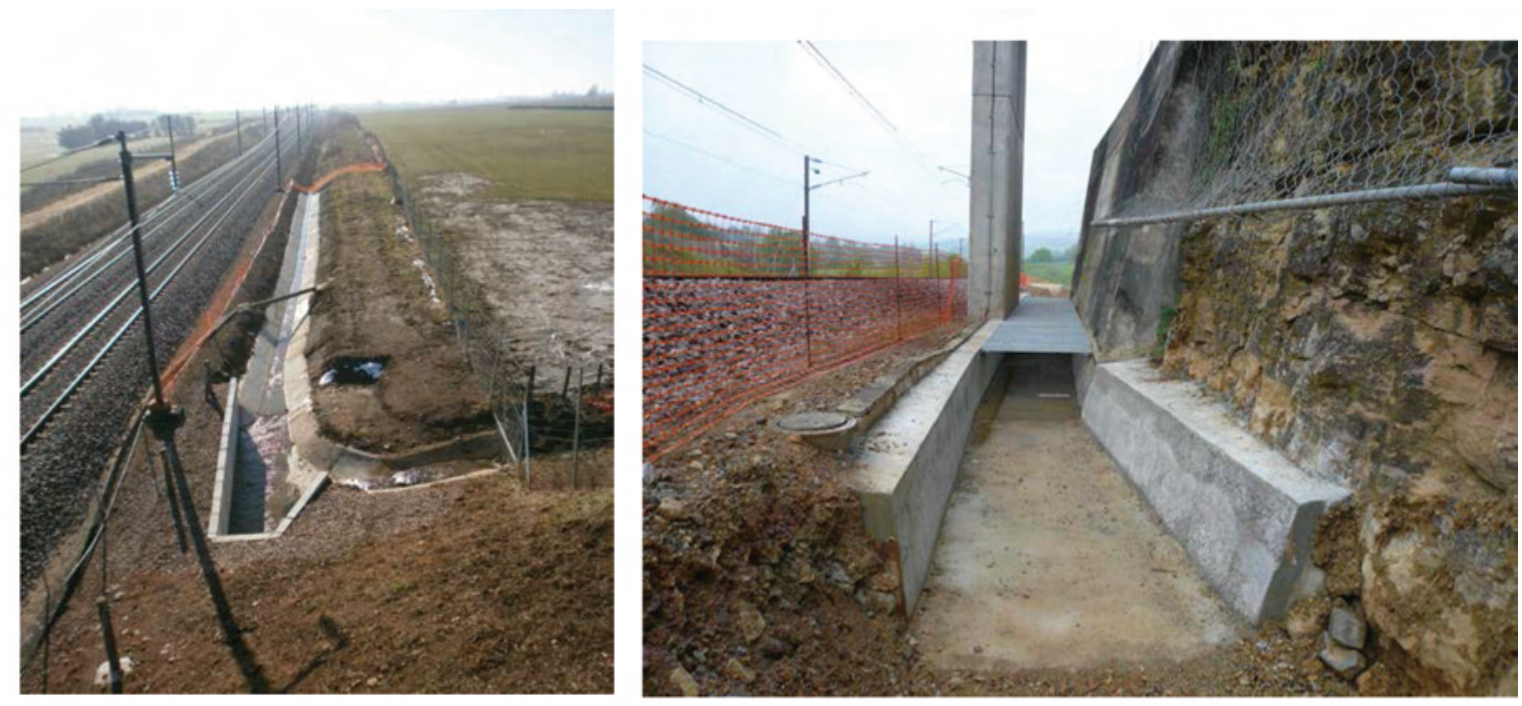

Figure 4 : Photos de travaux de régénération aux abords des voies $L G V$. 


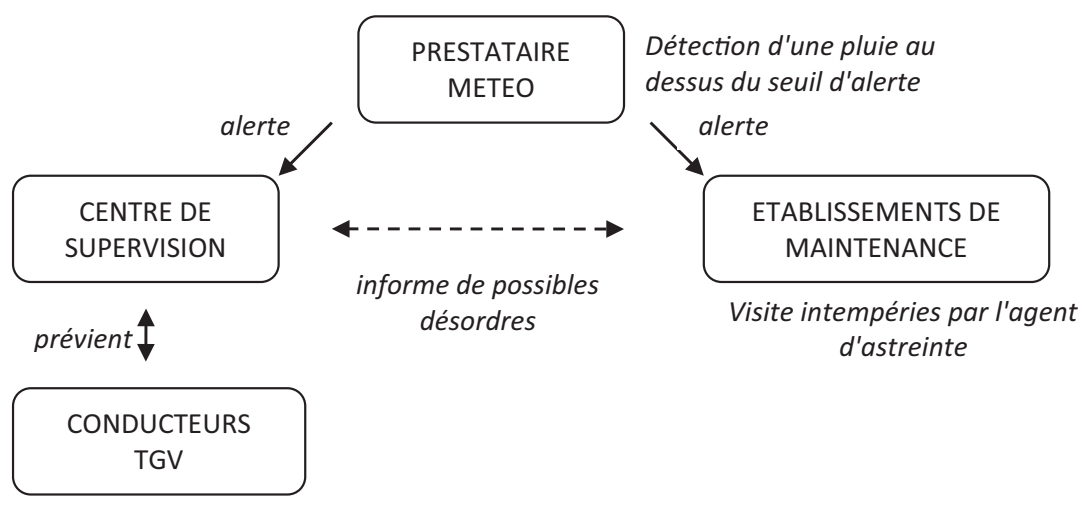

Figure 5 : Gestion de la surveillance météo vis-à-vis des circulations ferroviaires.

retour de leur part pour déterminer l'état de l'infrastructure. En parallèle, l'agent d'astreinte de l'établissement de maintenance doit effectuer une tournée intempéries dans le secteur. Cette tournée a pour but de vérifier l'état des drainages, de la voie et des installations ferroviaires pour en rendre compte au centre de supervision.

\section{III.3. Améliorer la connaissance du risque}

\section{III.3.1. Les limites des modélisations numériques pour le "risque Sarry"}

Aujourd'hui, les calculs par modélisation numérique sont courants et la méthode est acquise. Un des aspects importants du « risque Sarry » qui n'est pas pris facilement en compte par une modélisation numérique est le transport solide, c'est-à-dire la détermination du moment et des conditions pour lesquelles le grain de ballast est emporté [Novak et al., 2012 ; Yalin et Da Silva, 2001].

En effet, pour reproduire ces évènements, il faudrait obtenir des mesures de débits ou de hauteurs d'eau, afin de pouvoir caler les modèles. Dans notre cas, ces données sont impossibles à obtenir car il s'agit d'aléas courts (de 20min à $1 \mathrm{~h}$ ) et lorsque l'agent d'astreinte arrive sur les lieux de l'incident, l'eau s'est déjà évacuée. De plus, les traces sont rapidement effacées par les travaux effectués dans l'urgence afin de permettre la reprise rapide des circulations.

Par conséquent, les limites des modélisations numériques pour le " risque Sarry » se situent dans la connaissance des phénomènes mis en jeu et ils ne permettent pas de comprendre les effets des écoulements sur la banquette de ballast. Toutefois, ces modèles mathématiques continueront d'être utilisés pour connaître la hauteur et la vitesse en piste pour une pluie donnée.

\section{III.3.2. Pourquoi un modèle physique ?}

Au vu des limites des modèles numériques, il a été décidé de construire un modèle physique à échelle réduite (1/3). Tout d'abord, le modèle physique permet de visualiser le phénomène de ballast emporté par les écoulements et de mieux comprendre les enjeux de ce risque [Froehlich, 2013 ; Jafarnejad et al., 2013].

De plus, une fois la maquette construite, toutes sortes d'essais sont envisageables. Dans notre cas, le choix de ne pas modéliser seulement l'impact de l'eau coulant sur la piste mais d'intégrer au cahier des charges des obstacles aux écoulements comme un massif de poteau caténaire, un rétrécissement de piste sous un pont-route et un regard de collecteur rend plus réaliste les résultats obtenus. En effet, ces obstacles aggravent le phénomène d'emportement de ballast.

Il a été donc été proposé à RFF (Réseau Ferré de France), le gestionnaire d'infrastructures, de réaliser un modèle physique afin de mieux définir :

$>$ Les phénomènes mis en jeu lors d'un débordement des drainages :

> Les critères d'emportement du ballast (hauteurs / vitesses) afin de mieux définir le risque ;

$>$ L'impact de 3 types d'obstacles (le massif de poteau caténaire, le regard de collecteur drainant et le rétrécissement de la piste sous un pont-route).

Ce Projet de recherche, appelé Impact sur le Ballast des Eaux de Ruissellement (IBER) a été financé par RFF et est piloté par la Direction de l'Ingénierie de la SNCF. Les essais sur modèle physique à l'échelle $1 / 3$ ont été réalisés au Laboratoire de Constructions Hydrauliques (LCH) de l’Ecole Polytechnique Fédérale de Lausanne (EPFL).

\section{III.3.3. Les résultats obtenus (LCH, 2011)}

Pour caractériser le ballast emporté par les eaux de ruissellement, trois classes de transport ont été définies avec les limites entre chacune (cf. Tableau 2). Le débit limite correspondant à la séparation entre deux classes de transport solide varie en fonction de la configuration (avec ou sans obstacle) et de la pente longitudinale de la piste.

Les essais ont été effectués pour des pentes longitudinales de $0,5 \%$ à $3,5 \%$.

\section{Résultats sans obstacle :}

Les essais sans obstacle (cf. Figure 6) ont permis de fixer un état de référence. Pour chaque pente, le comportement de la banquette de ballast a été analysé et une hauteur d'eau ainsi que le débit correspondant, séparant les classes de débit solide a été identifié. Ceci permet de quantifier le risque. Les conditions d'écoulement produisant une certaine classe de transport solide ont été identifiées en l'absence d'obstacle, pour l'état de référence et ensuite pour chaque type d'obstacle.

En l'absence d'obstacle, des courbes délimitant les classes de transport peuvent être définies en fonction de la hauteur d'eau et de la vitesse de l'écoulement. Il semble néanmoins plus pertinent de définir des relations entre hauteur d'eau et pente longitudinale afin de délimiter les classes en absence d'obstacle. En effet, la vitesse de l'écoulement mesurée peut varier selon la position verticale de la mesure (profil de vitesse) et un lien direct entre vitesse, hauteur et pente existe et est donné par l'équation de Manning-Strickler. Les classes 
Tableau 2 : Définition des classes de transport solide et limites associées.

\begin{tabular}{|c|c|}
\hline Classe de transport & Limite \\
\hline Pas de transport solide & \\
\hline \multirow{2}{*}{ Début du transport } & Limite 0 \\
\hline & \multirow[b]{2}{*}{ Limite 1} \\
\hline \multirow{2}{*}{ Transport linéaire } & \\
\hline & \multirow[b]{2}{*}{ Limite 2} \\
\hline Rupture de la géométrie de la banquette, transport intense & \\
\hline
\end{tabular}

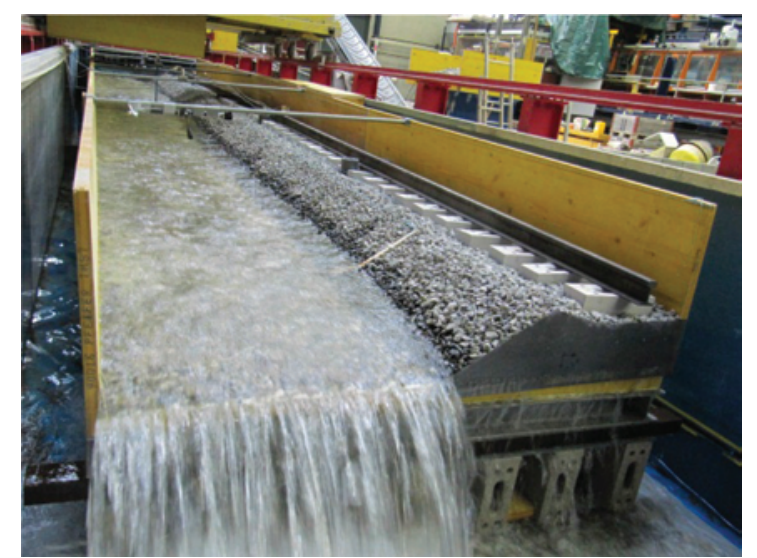

Figure 6 : Maquette à l'échelle 1/3, avec écoulement en piste sans obstacle.

de transport solide peuvent également être délimitées en fonction du débit et de la pente.

Dans un premier temps, les résultats obtenus montrent que : $>$ Des critères fixes de hauteur d'eau et vitesse, quelle que soit la pente, ne sont pas pertinents.

$>$ Pour des pentes faibles, les critères purement empiriques utilisés jusqu’à présent sont plus restrictifs. Une optimisation des études et des travaux de régénération est donc possible.

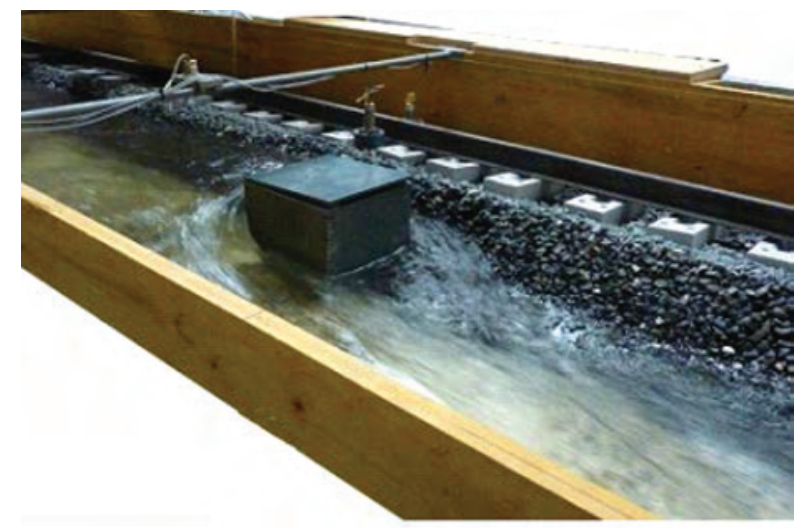

Figure 7 : Vue du phénomène autour d'un regard de collecteur drainant.
$>$ Pour de fortes de pentes, le ballast risque d'être emporté dès quelques centimètres d'eau.

Résultats avec obstacles :

En présence d'obstacles, il est moins aisé d'identifier des classes en fonction de la hauteur d'eau. La présence d'obstacles crée une courbe de remous à l'amont de ces derniers. De plus, la hauteur d'eau le long de l'obstacle à l'amont est clairement plus élevée que celle à l'aval. Il a ainsi été décidé de délimiter les classes de transport en fonction de la pente longitudinale et du débit liquide. Un coefficient d'impact d'obstacle (CIO) qui est défini comme étant le rapport entre le débit spécifique limite en absence d'obstacle et le débit spécifique limite pour un obstacle donné, a ainsi pu être défini pour chaque obstacle, pour un type de piste donné.

Une fois les équations limites établies pour chaque obstacle, le CIO a été défini. Pour chacun des obstacles, trois CIO sont définis, un par limite de classe de transport.

$>$ Si le CIO > 1, l'obstacle augmente l'érosion par rapport à une situation sans obstacle.

> Si le $\mathrm{CIO}<1$, l'obstacle a un effet plutôt stabilisant.

La présence d'un regard de collecteur drainant (cf. Figure 7) accentue la mise en mouvement du ballast pour des faibles pentes, par rapport au cas sans obstacle. Par contre, pour des fortes pentes longitudinales, le regard a un impact plutôt stabilisateur.

La présence d'un poteau caténaire accentue la mise en mouvement du ballast, quelle que soit la pente. Mais son impact diminue pour des fortes pentes.

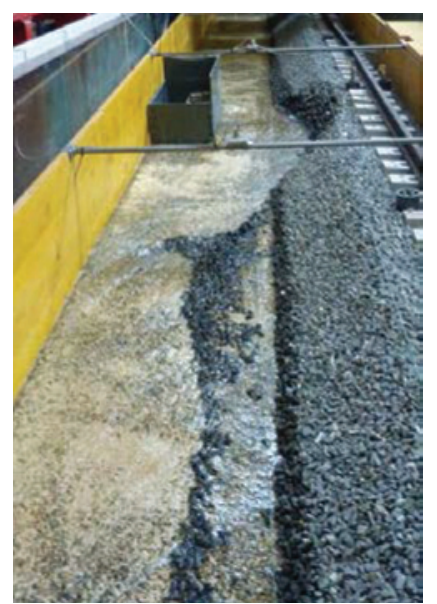

Figure 8 : Impact sur la banquette de ballast des écoulements sur la piste rétrécie sous un pont-route. 
L'impact de la pile de pont (cf. Figure 8) est constant quelle que soit la pente longitudinale. Cet obstacle est le plus critique, il a en effet le CIO le plus élevé. De plus, pour chaque obstacle les zones d'érosion locales ont pu être identifiées.

\section{CONCLUSION}

Les résultats obtenus grâce au modèle physique peuvent être appliqués immédiatement sur toutes les études et les expertises quels que soient la ligne et le réseau ferré. Ils permettront une optimisation des travaux à envisager.

De plus, ils ont été filmés et sont donc utilisables pour toute la communication autour du risque de ballast emporté par les écoulements, de ses enjeux, des phénomènes hydrauliques et des protections envisagées.

Sur le réseau ferré national, ils permettront de mieux définir les zones à risques par intégration de ces résultats dans les diagnostics en cours.

Ces résultats trouvent immédiatement leur place :

> en Ingénierie de Maintenance (optimisation de la régénération),

$>$ en Ingénierie de Projet (intégration et explicitation du risque lors de la conception),

$>$ en Ingénierie Système (optimisation de la surveillance météo).

Cette démarche d'analyse de risques a permis d'appréhender une problématique de l'origine de l'aléa jusqu'à l'optimisation des travaux et de la surveillance des installations. Les enjeux de sécurité ferroviaire et de régularité étant très forts, notamment sur LGV, ce travail a permis de faire prendre conscience des impacts d'un événement pluvieux court et intense sur l'ensemble du système ferroviaire.

\section{REFERENCES}

Froehlich D. C. (2013) - Sizing loose rock riprap to protect stream banks. doi: 10.1002/rra.1587. River Res. Applic. 29 : 219-235

Jafarnejad M., Franca M. J., Pfister M., Schleiss A.J. (2013) - Time Dependent Failure Analysis of Compressed Riprap as Riverbank Protection. 35th IAHR World Congress, Chengdu, China, 9-13 September 2013

LCH (2011) - Impact sur le Ballast des Eaux de Ruissellement (IBER) - Etude sur modèle physique. Rapport d'étude LCH $N^{\circ}$ 13/2011, non-publié. 13

Novak P., Guinot V., Jefreey A., Reeve D.E. (2010) — Hydraulic modelling - an introduction: principles, methods and applications. Spon Press: Oxon. 599 p

Nivon D., Pams Capoccioni C. (2011) - Analyse du risque hydraulique sur lignes à grande vitesse exploitées. Symposium international Géorail. 1 : 749-755

Pams Capoccioni C., Nivon D., Chenot L. (2012) - Les crues à grande vitesse. Revue Générale des Chemins de Fer. 221 : 100-106

Pams Capoccioni C., Nivon D., Amblard J., De Cesare G., GhIlARDi T., JAFARNEJAD M. (2013) - Analysis of ballast transport in the event of overflowing of the drainage system on high speed lines. Railway Track Science and Engineering Ballast : Issues and Challenges. 1 : 52

Perlet J. (2002) — Les aménagements hydrauliques de la Ligne TGV Méditerrannée. Revue Générale des Chemins de Fer. 103

YAlin M.S., DA Silva A. M. (2001) - Fluvial processes Monograph, IAHR, Delft. 197 p 